\title{
Panel Discussion on Inducer Design Criteria
}

\author{
Marc Wegner \\ Centre de Recherche et d'Essais de Machines Hydrauliques de Grenoble (CREMHyG), France
}

\author{
Allan J. Acosta \\ California Institute of Technology, USA \\ Yoshinobu Tsujimoto \\ Department of Engineering Science, Osaka University, Japan
}

This article reports a panel discussion titled Inducer Design Criteria presented at the 9th International Symposium on Transport Phenomena and Dynamics of Rotating Machinery (ISROMAC-9). The presentations of the panelists and the subsequent discussions are summarized. It is shown that cavitation instabilities are major problems in modern turbopumps and that design criteria to eliminate them are needed. Available design methods for inducers and marine propellers are reviewed, and new criteria to enhance stability are proposed. The current status of CFD is reviewed and an example of successful application is shown. Discussions of several specific topics are reported and future research needs are noted.

Keywords Cavitation, CFD, Design, Inducer, Instability

Cavitation instabilities are becoming a major concern in modern turbopump inducers for rocket engines. In the past, impeller inducers have been designed with the main focus on maximizing the suction performance. Currently, there is a demand to establish a design criterion that avoids the cavitation instabilities while maximizing the suction performance. Under these

Received 25 June 2002; accepted 1 July 2002.

The authors thank all the panelists and the discussers as well as the chair of the panel, Professor Joseph Katz, who not only participated in the panel but also reviewed the draft of this manuscript. Special thanks to Mr. Thomas Zoladz of NASA MSFC who kindly offered to transcribe the discussions on which this report is based. Thanks also to Mr. Koichi Yonezawa and Mr. Akira Fujii of Osaka University for their assistance in recording the panel discussion and preparing the manuscript.

Address correspondence to Yoshinobu Tsujimoto, Department of Engineering Science, Osaka University, Osaka, 560-8531, Japan. E-mail: tsujimoto@me.es.osaka-u.ac.jp

circumstances, a panel discussion titled Inducer Design Criteria was organized by the first two authors and held on February 12, 2002, at the ISROMAC-9 meeting, which was organized by the last author, in Honolulu, HI. It was preceded by sessions titled "Free Surface Flows and Cavitation" and "Rocket Turbopumps," in which many related technical papers were presented. The panel discussion was intended to clarify (1) what is needed, (2) what is available, and (3) what is being done, and had the ultimate goal of setting the direction of research and development. Chaired by Professor Joseph Katz of Johns Hopkins University, presentations were made by seven panelists from space development agencies, industry, and universities and were followed by discussions from the floor. This short article has been prepared for the purpose of recording the presentations and discussions so that we can share the outcome with the engineers and researchers who are interested in this field but could not join the panel. (Numbers such as $F D-A B S-137$ refer to technical papers in ISROMAC-9. Most of them will be or have been published in the International Journal of Rotating Machinery).

\section{SUMMARY OF PRESENTATIONS}

\section{Marc Wegner}

Affiliated with CREMHyG, France (Maboral@aol.com) Marc Wegner speaks in parallel to paper FD-ABS-137.

First, the averaged axial pressure distribution measured on an inducer casing is shown and the relationships with the instability are discussed, focusing on the axial pressure gradient. Figure 1 shows the pressure distribution plotted against the distance from the leading edge, represented as the percentage of the total axial length, for various flow rate ratios $Q / Q_{n}$. At higher flow rates, the pressure gradient appears to be very small at the inlet section and the overlapping section. However, no rotating cavitation occurs because the cavity volume remains rather small. At lower flow rates, the axial pressure slope is steeper, and backflow 


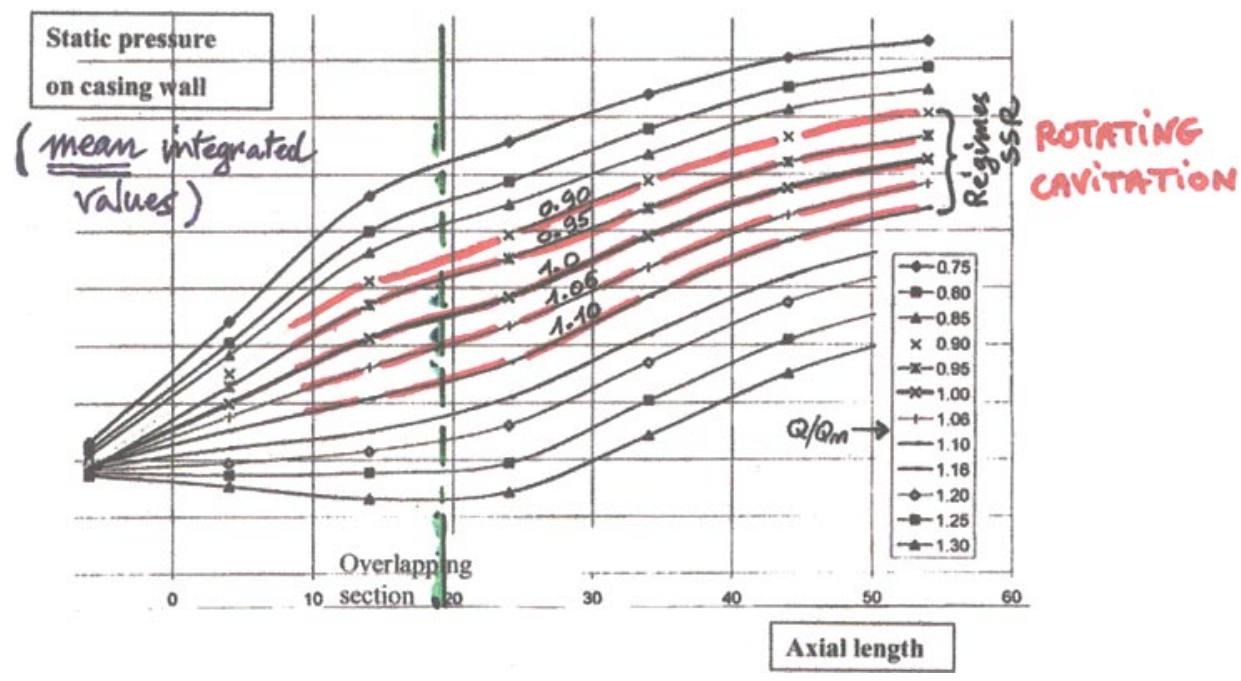

FIGURE 1

Axial pressure increase in an inducer.

begins without rotating cavitation. In fact, rotating cavitation is found at intermediate flow rates, when pressure gradient is low with sufficient amount of cavity volume. So one interesting question for the designer is: What kind of flow should be chosen for the overlapping section? Or, in other terms: What should be the value of the ratio between nominal flow and zero-incidence flow to avoid rotating cavitation?
Next, two examples focus on the ratio of the inlet incidence angle to the blade angle. It is confirmed that rotating cavitation occurs in the region of intermediate incidence values.

Further, it is pointed out that at a lower flow rate, backflow begins, which could be an improvement, from the stability viewpoint. Figure 2 shows a schematic view of the internal inducer cascade flow at the blade tip. At the outer radius, a source region

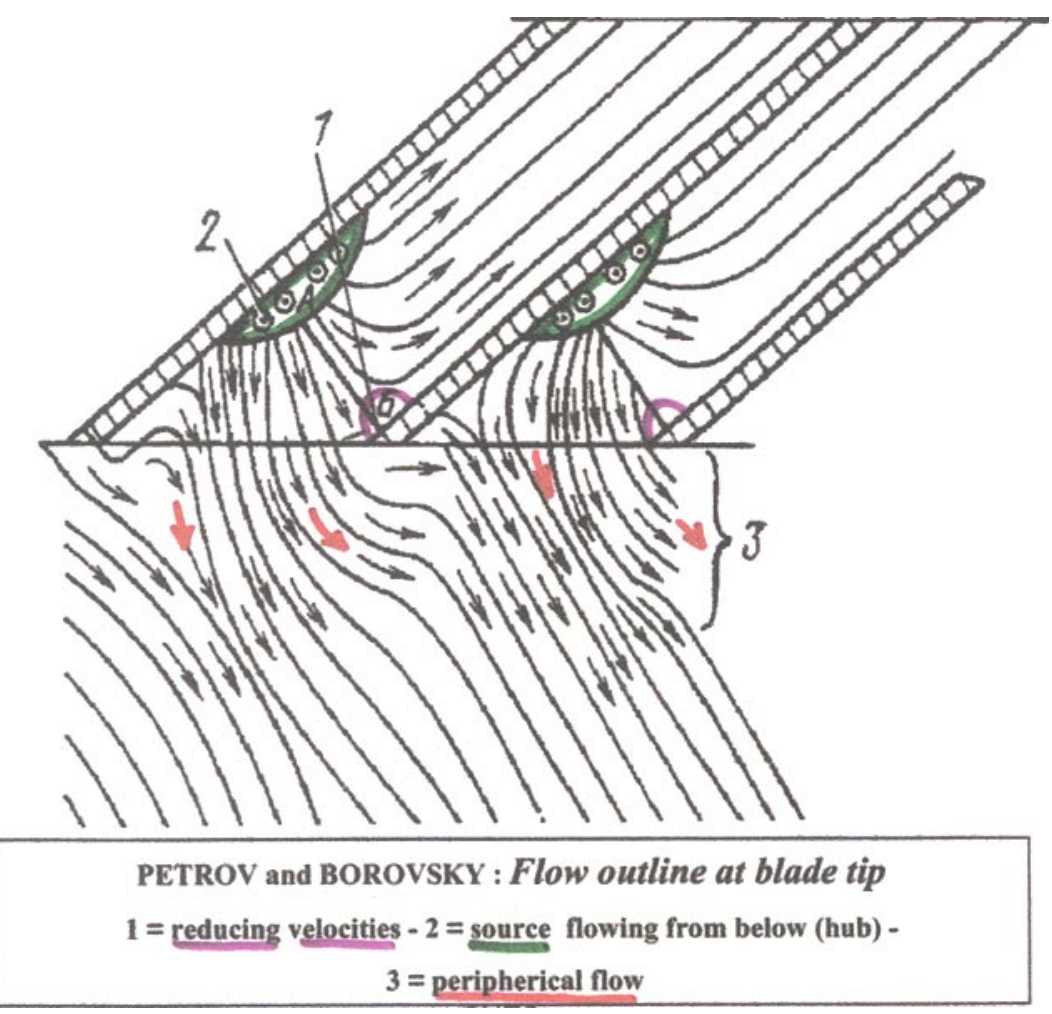

FIGURE 2

Flow outline at blade tip. 


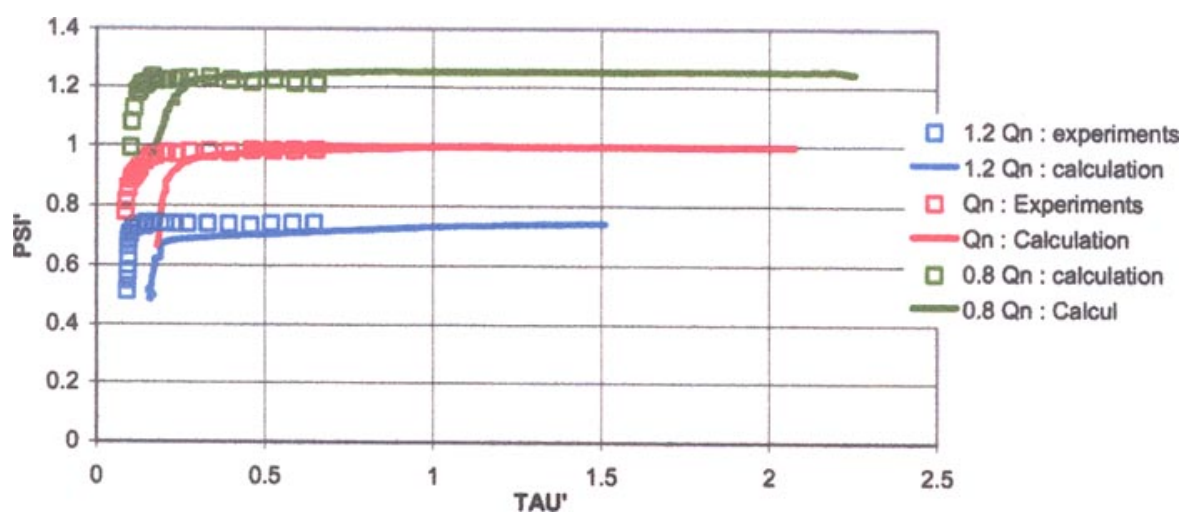

FIGURE 3

Comparisons between numerical and experimental head-drop charts.

at the starting point of the overlapping section and on the suction surface of the blades is induced by radial flow from the hub section. Radial flow on the suction side can be calculated by Navier-Stokes codes and may be observed, as well.

From the point of view of stability, the main result of source occurrence on the suction side is to increase local pressure and therefore to avoid a negative pressure gradient and rotating cavitation.

\section{Jean-Luc Reboud}

Affiliated with LEGI-Grenoble and LTDS/ENISE, France (reboud@enise.fr) Jean-Luc Reboud speaks in parallel to paper FD-ABS-127 and paper B8.002 at Cav2001, available from http://cav2001.caltech.edu/.

Results of quasisteady three-dimensional cavitating flow calculations based on paper FD-ABS-127 and unsteady twodimensional cavitating flow calculations based on paper B8.002 are introduced (all two-dimensional and three-dimensional computations were performed at LEGI-Grenoble by the turbomachinery and cavitation team). They are both based on a single fluid model in which the fluid density is assumed to change largely near the saturation pressure.

The three-dimensional quasi-steady calculations can predict the noncavitating performance and the cavitation breakdown nicely, as is shown in Figure 3. The relationship between the head breakdown and the development of cavities is discussed by the panel, as is the quantitative discrepancy in the head breakdown point.

The two-dimensional unsteady calculations with multiple blades succeed in predicting rotating cavitation (Fig. 4). Suction performance is compared with the results of single-channel computations and the necessity of multiple-channel calculations is clarified.

All of these results show that the CFD with cavitation has made significant progress in the past couple of years and is becoming a useful tool for design.

\section{Akinori Furukawa}

Affiliated with Kyusyu University, Hakozaki, Fukuoka, Japan (fmfuru@mech.kyushu-u.ac.jp), Akinori Furukawa speaks about a short note prepared for the panel.

A graphical method of estimating required NPSH of a whole pump with an inducer is introduced. Once we know the suction performances of the main pump and the inducer, the suction performance of the whole pump can be obtained graphically.

It is shown that the head $\psi$ of various inducers can be correlated with a flow rate ratio at the outlet $m_{2}=\phi_{2} / \tan \beta_{b t 2}$, where

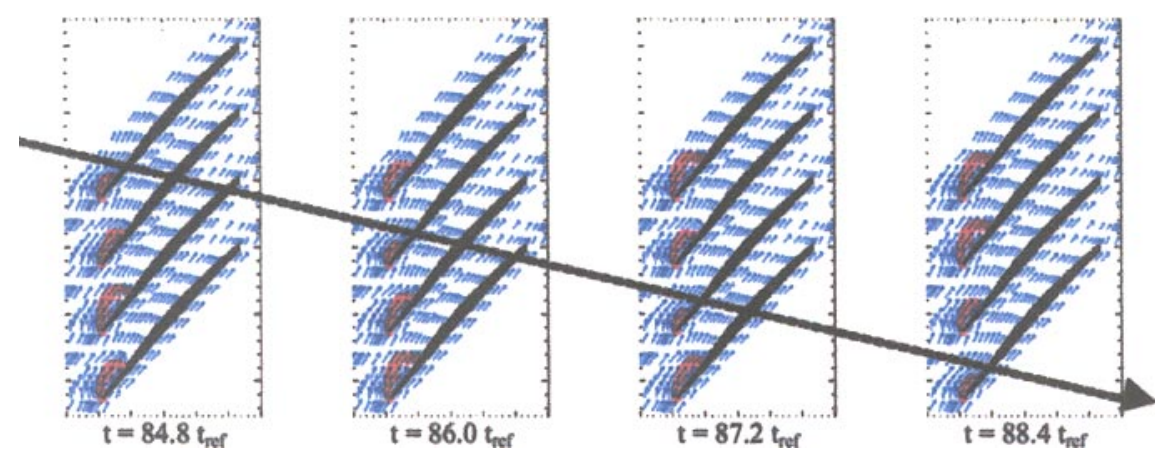

FIGURE 4

Rotating cavitation simulated by a numerical method. 


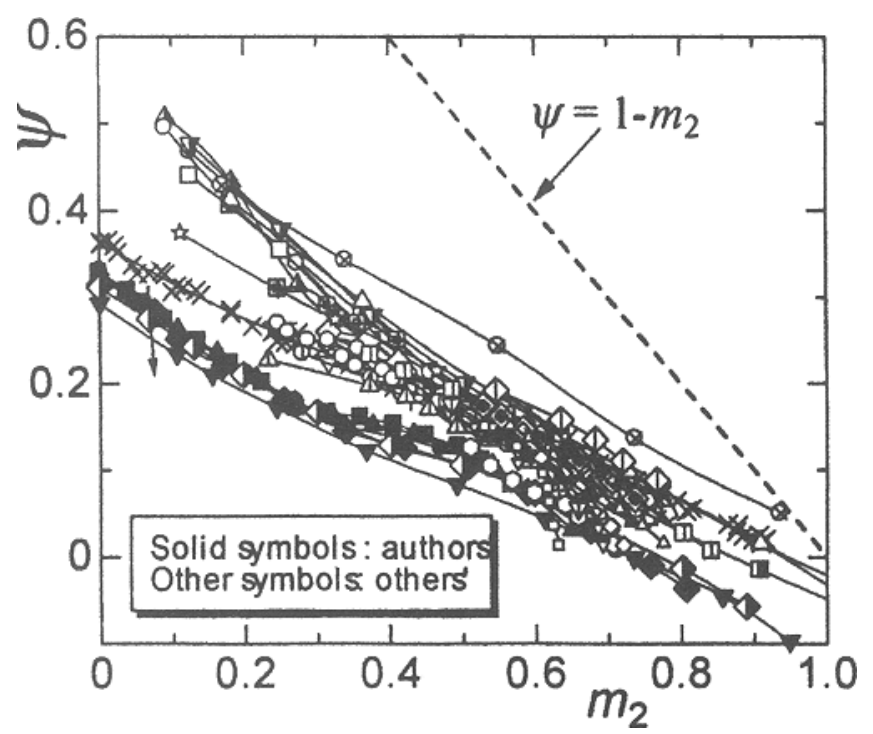

FIGURE 5

Head-rise characteristics of inducer.

$\phi_{2}$ and $\beta_{b t 2}$ are the flow coefficient and the outlet blade angle, respectively (Fig. 5). On the other hand, the cavitation number for 50\% head-drop, $\tau=N_{P S H_{50 \%}} /\left(U_{t 1}^{2} / g\right)$, can be correlated with the inlet flow rate ratio $m_{1}=\phi_{1} / \tan \beta_{b t 1}$, as shown in Figure 6. Effects of other design parameters on these correlations were discussed in the panel. These correlations are useful for the basic design of inducers.

The region of cavitation surge is also shown, in a map similar to Figure 6, and the effects of the blade inlet angle are discussed.

\section{Spyros A. Kinnas}

Affiliated with the University of Texas, Austin, TX (kinnas@ mail.utexas.edu), Spyros A. Kinnas speaks in relation to "An

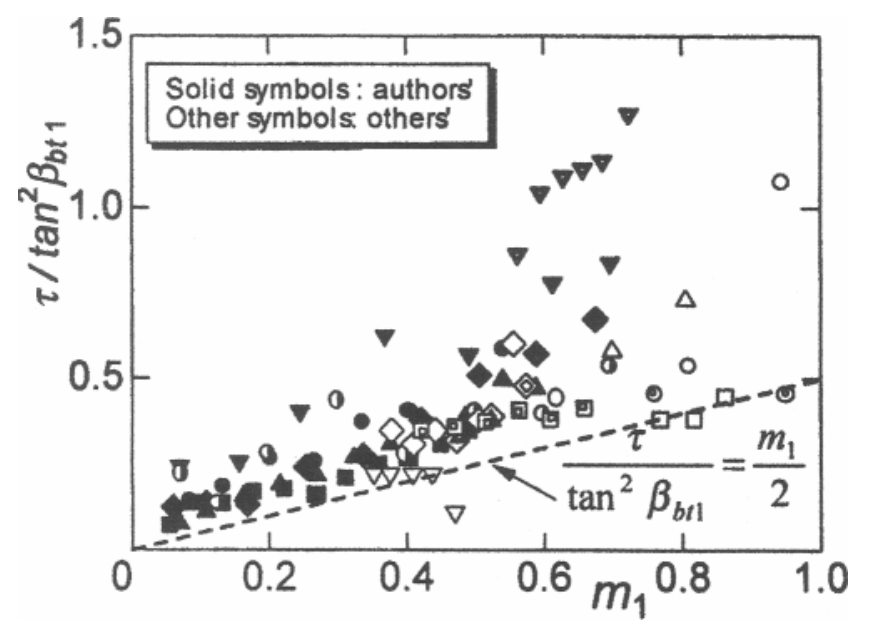

FIGURE 6

NPSH of 50\% deterioration in inducer head-rise.
Integrated Computational Technique for the Design of Propellers with Specified Constraints on Cavitation Extent and Hull Pressure Fluctuation," by Kinnas and colleagues, which was presented at the International CFD Conference, 5-7 June 1999, Ulsteinvik, Norway.

The design of cavitating marine propulsor blades is discussed. First, various types of propulsors are introduced and propeller terminology is explained, as contrasting with pump terminology. The design target is not only to maximize propulsion efficiency but also to minimize pressure fluctuation, blade erosion, and unsteady forces on the hull and blades. With a smaller blade area, we have more cavitation but higher efficiency and smaller frictional forces. So the final design must be a compromise, and an automated optimization process is required (Fig. 7, from the CFD'99 paper). Such a design system, CAVOPT-3D, is introduced. This system includes three-dimensional unsteady cavitating flow analysis. Unsteady features such as hull pressure fluctuation, unsteady cavitation extent can be taken into account in the design optimization (Fig. 8). The importance of structural constraint on the selection of maximum blade thickness and skew is stressed.

\section{Thomas Zoladz}

Affiliated with NASA Marshall Space Flight Center (Thomas.F.Zoladz@msfc.nasa.gov), Thomas Zoladz speaks about instabilities.

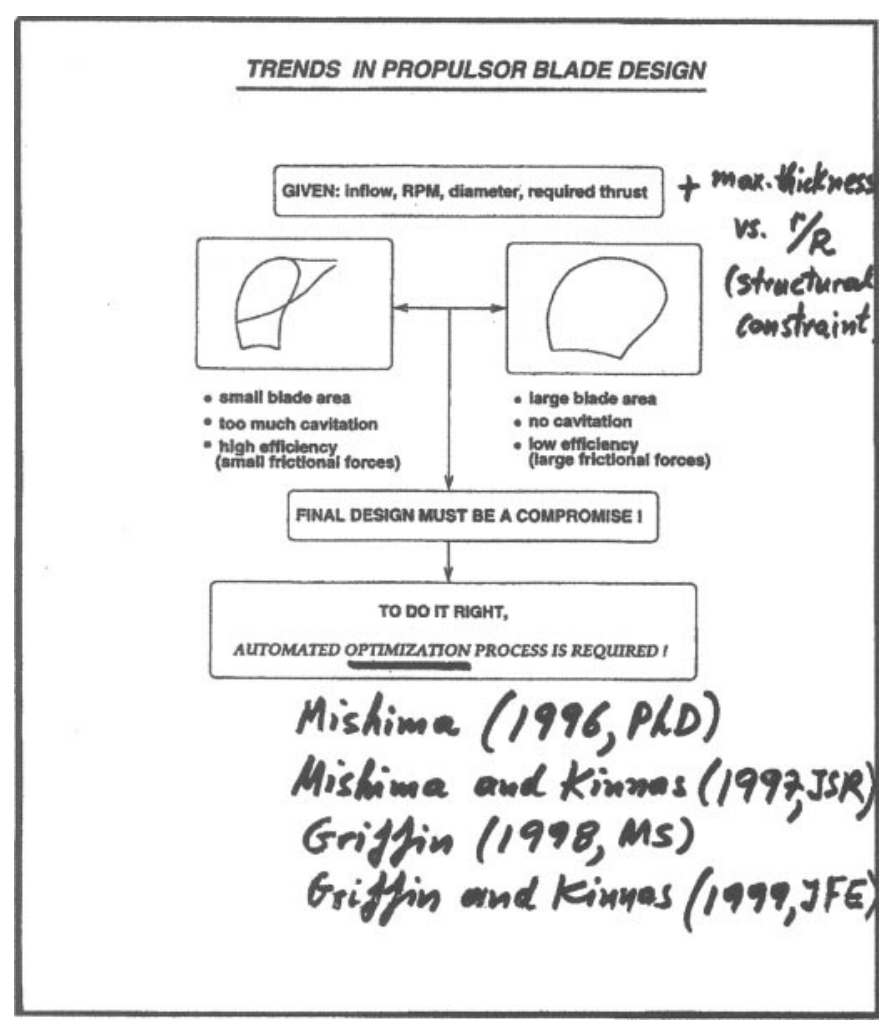

FIGURE 7

Trends in propulsor blade design. 


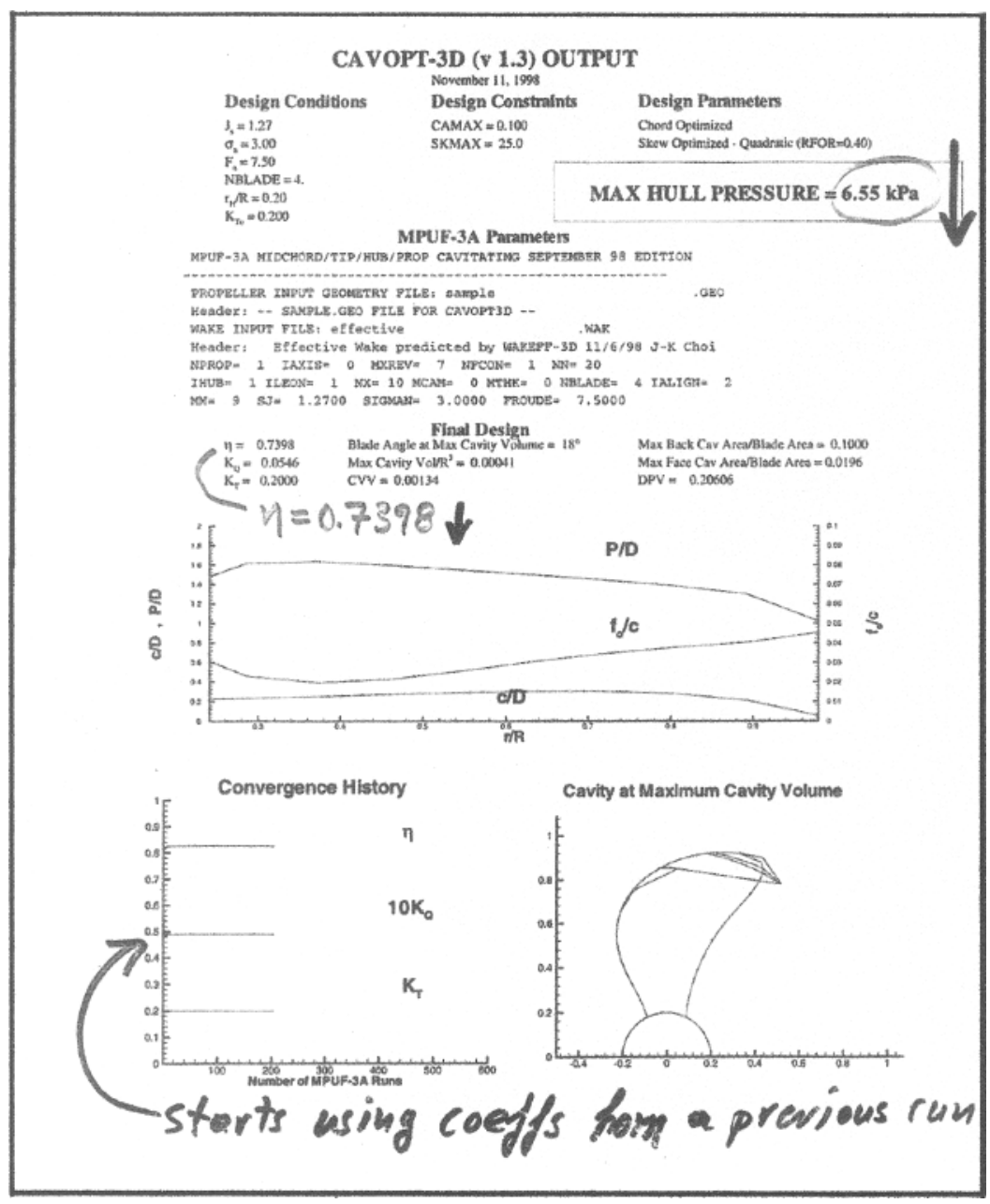

FIGURE 8

CAVOPT-3D (v1.3) output.

Various kinds of cavitation instabilities observed in the Space Shuttle Main Engine (SSME) high-pressure oxygen turbopump (four-bladed) are reported (Fig. 9). Their cell structure, covering the number of cells from 0 , surge mode, to forward rotating and backward rotating, is identified from the on-blade strain and housing pressure measurements. They occur at smaller suctionspecific speeds than does alternate blade cavitation. The second example is a surge mode oscillation with the frequency $3(\mathrm{rc}-\mathrm{N})$ (rc, rotating cavitation frequency; $\mathrm{N}$, shaft rotational frequency), which was observed in the Fastac 60K engine oxygen turbopump inducer (three-bladed; Fig. 10). This component appears to be associated with rotating cavitation and is important because this component may cause strong pump-facility coupling.

The importance of the characterization of loads induced by the various forms of cavitation-induced instabilities and of the measurement of cavitating turbopump dynamic transfer function is stressed. (Refer to T. Zoladz, "Observations on Rotating Cavitation and Cavitation Surge from the Development of the Fastrac Engine Turbopump," AIAA 200-3403, the 36th
Joint Propulsion Conference, Huntsville, AL, 17-19 July, 2000.)

\section{Takashi Shimura}

Affiliated with the National Aerospace Laboratory of Japan (shimura@kakuda-splab.go.jp), Takashi Shimura speaks in parallel to paper FD-ABS-111.

Two examples of cavitation instabilities observed in the development of HIIA turbopump inducers are presented. The first one is a rotating-stall type of instability, which appears to be associated with the sudden head decrease (Fig. 11) observed with the original design of LE-7A LH2 turbopump inducer. It has one cell and the pressure pattern rotates at a rotational speed about half of that of the impeller. This instability occurs only with liquid hydrogen and not with water. Details of this instability are reported in paper FD-ABS-111.

The second instability is an attached (uneven) cavitation, observed in the LE-7A LOX turbopump inducer, which causes unacceptable synchronous vibration of the shaft (Fig. 12). The 


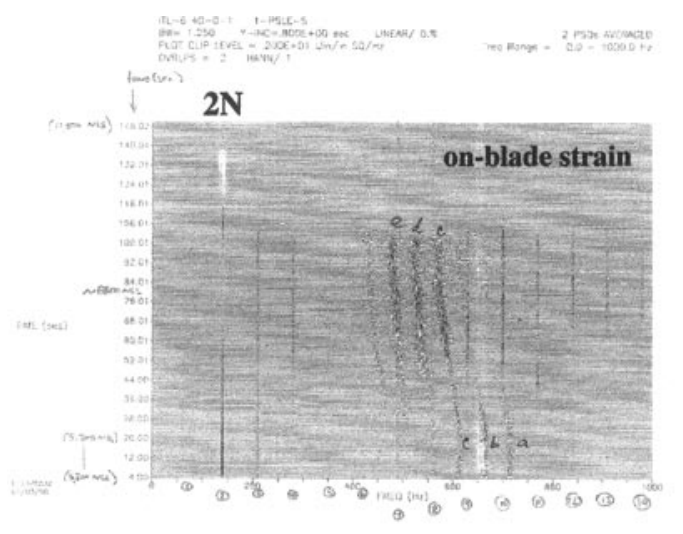

- SSME High Pressure Oxygen Turbopump (HPOTP) inducer cavitation study initiated in mid 90's to support high synchronous vibration

- Inducer (4-bladed) water flow testing mapped both rotating and attached cavitation (hsng p', blade strain)

- Coexisting multi-cell flow field preceded attached alternate blade cavitation:

$\begin{array}{lll}\text { comp. } & \text { Nss } & \text { \#cells(+with shaft) } \\ \text { a } & 5,300 & -1 \\ \text { b } & 5,300 & \text { non-rotating } \\ \text { c } & 7,600 & -3 \\ \text { c' } & 7,600 & +1 \\ \text { d } & 7,600 & -2 \\ \text { d' } & 7,600 & +2 \\ \text { e } & 9,000 & +3\end{array}$

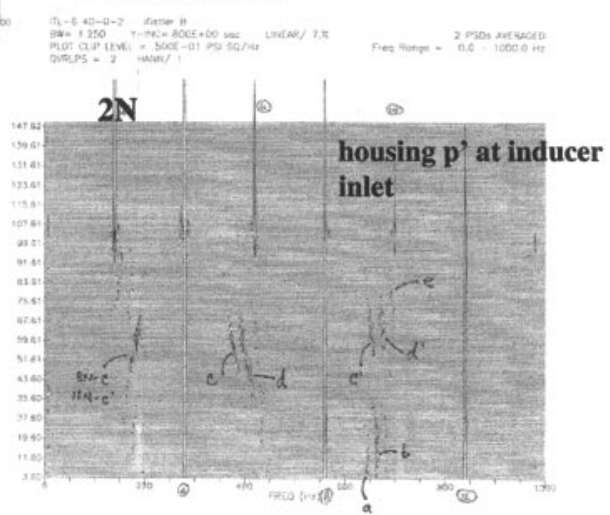

FIGURE 9

Spectrums of strain and inlet pressure fluctuation of SSME HPOTP.

magnitude of shaft vibration depends on small manufacturing errors and also on residual unbalance. This instability is harmful also to the blade since the blade of the cavity attachment shifts occasionally during operation.

\section{Kazuyoshi Miyagawa}

Affiliated with Mitsubishi Heavy Industry, Takasago R\&D Center, Takasago, Japan (miyagawa@wb.trdc.mhi.co.jp) Kazuyoshi Miyagawa speaks about new inducers.

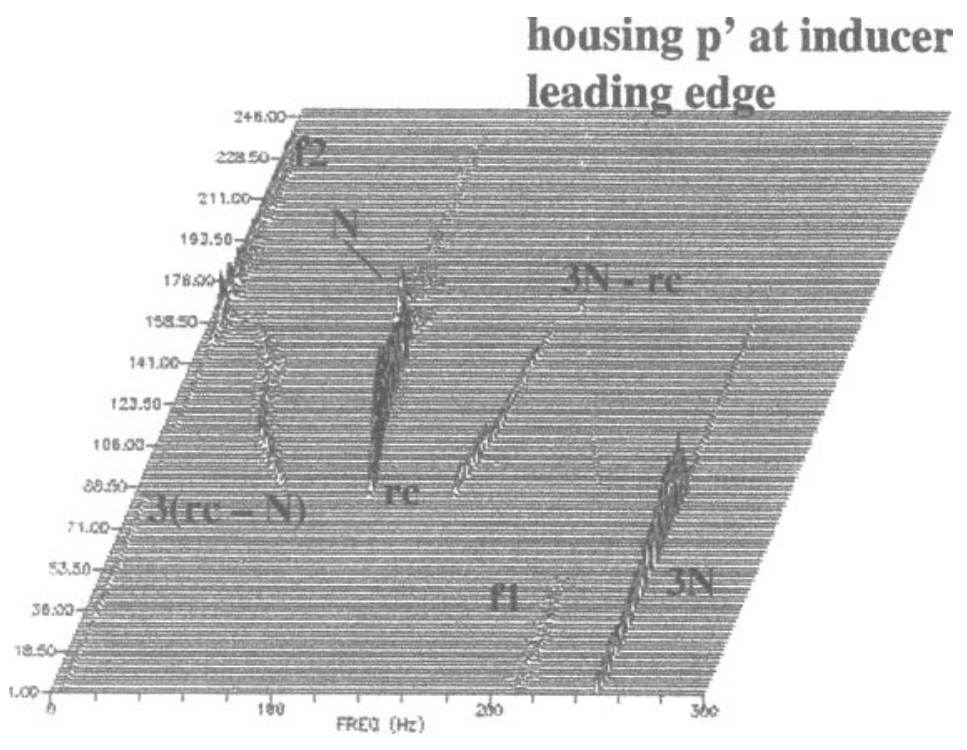

FIGURE 10

Spectrum of inlet pressure fluctuation of Fastac $60 \mathrm{~K}$ engine oxygen turbopump inducer. 


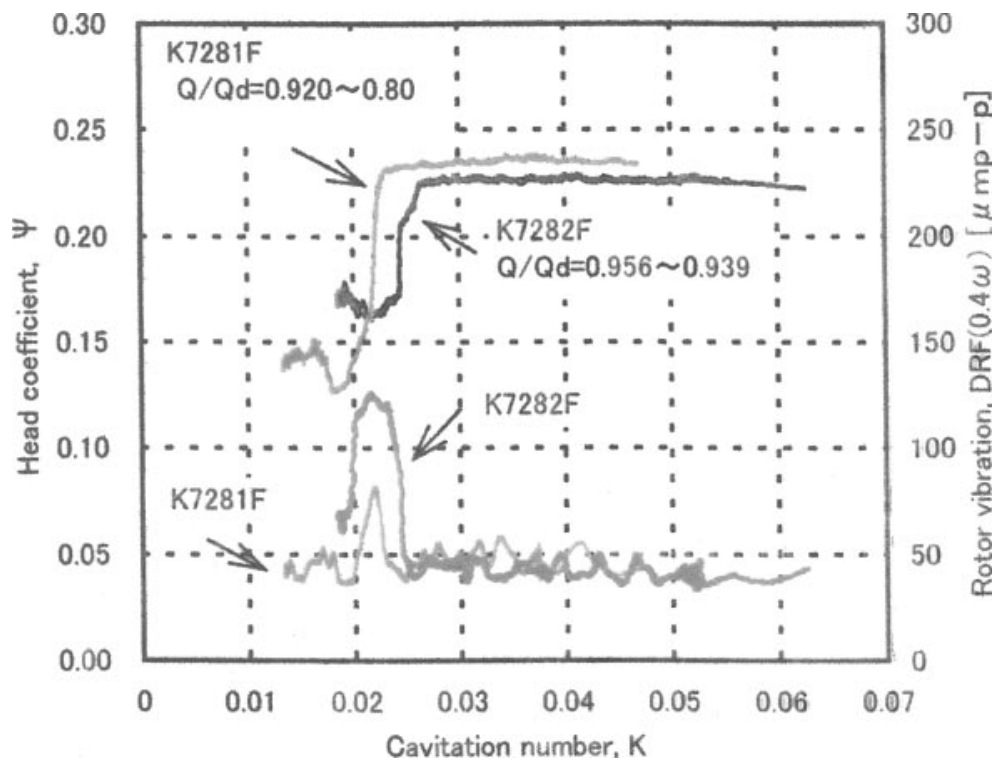

FIGURE 11

Suction performance and rotor vibration of LE7A LH2 inducer.

A new design for inducers with less cavitation damage, for application to heavy-duty multistage centrifugal pumps such as those used in power plants, is introduced. To reduce the damage due to tip leakage cavitation, the outer part of the impeller is tilted backward, to the pressure side (Fig. 13), keeping other design parameters, such as blade's leading and trailing edge angles, the same. A noncavitating CFD code is extensively used for the design.
From noise and cavitation intensity measurements and erosion tests, it has been proved that the durability of the newly designed inducer is a significant improvement over the original helical design. Visual observation shows that the tip leakage cavitation and the cloud cavitation following the tip leakage cavitation are mitigated by the new design (Fig. 14). The required NPSH is improved by $6 \%$, with the penalty of $2 \%$ head decrease.

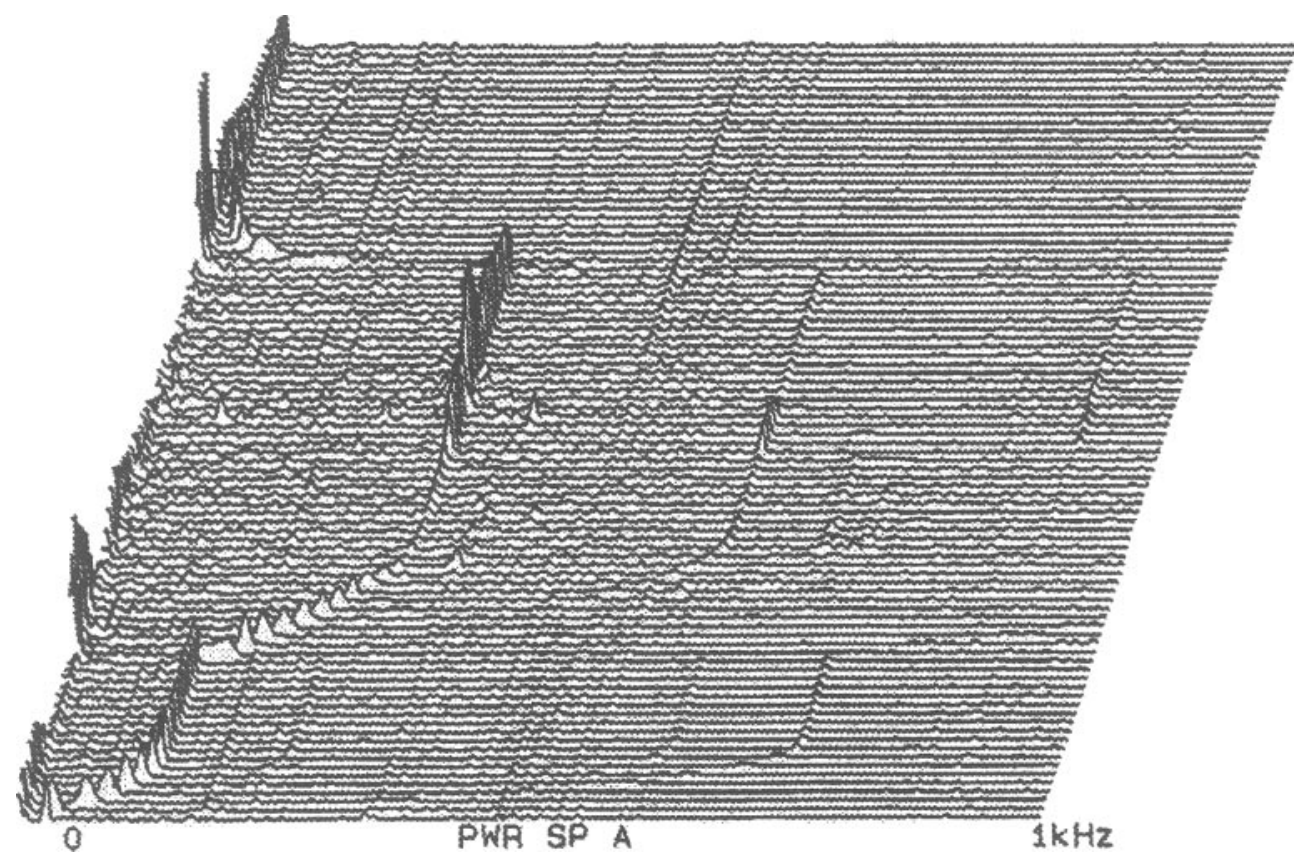

FIGURE 12

Spectrum of rotor vibration, LE7A LOX turbopump inducer. 
1. Original type (flat helical type)

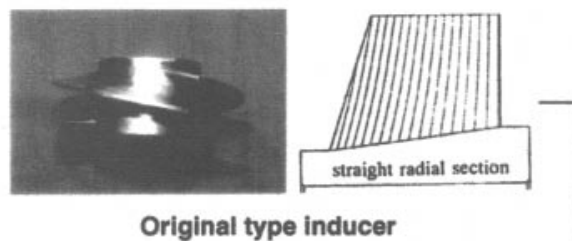

2. Modified type (ogival leading edge)

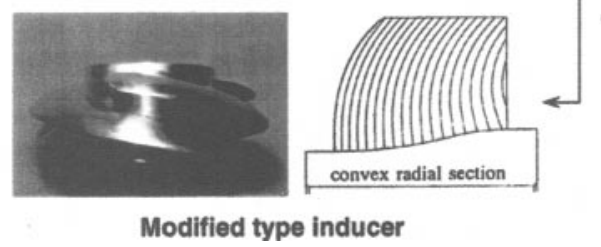

\section{Concept}

- Changing radial section profile

- Same in the design parameter (eq. inlet, outlet angle etc.)

- Reduce leading edge tip

section cavitation

FIGURE 13

Inducer design to avoid cavitation damage.

\section{SUMMARY OF DISCUSSIONS}

There was a round robin of questions from the floor and answers from the presenters, but the recorded voice data were insufficiently accurate to make a verbatim transcription for publication, so the following section is the authors' edited copy of the recording combined with our own memory of the discussions. The individuals asking the questions and giving the responses are not identified, as there was some uncertainty about their identities. Those involved in the discussion were Luca d'Agostino (University of Pisa); Christoph Bonhomme (CNES); Philippe Dupont (Sulzer, Winterthur, Switzerland); Robert Hendricks (NASA LRC, Cleveland, OH, USA); and David Japikse (Concepts NREC, Norwich, VT, USA). The topics of discussion were found to fall basically into three categories: the effect of leadingedge sweep; the integral design of an inducer (the cavitating part of the pump) and the main stage; and the role of cavitating instabilities and their design criteria in pump design.

\section{Effect of Sweep}

First, the favorable effect of forward sweep on suction performance was pointed out. Since the structure causes high stress at the root in that case, it is usually used in combination with a rotating shroud, which may cause high drag. Considering the velocity component in a plane normal to the leading edge, it can be shown that the effective cavitation number is increased by a factor $(1 / \sin \alpha)$, where $\alpha$ is the angle between the leading edge and the circumference. This rule applies well for forward and backward sweep when the amount of the sweep is small and, hence, the effect of the sweep is the same for forward and backward sweep. However, when the amount of sweep is large, the effect of secondary flow becomes larger; we have smaller or larger backflow at the inlet for forward- or backwardswept inducers. Probably associated with this effect is the shorter cavity at the tip and the longer blade-surface cavity at the inner radius, resulting in better suction performance by forward-swept inducers. Rotating cavitation can be suppressed by backward sweep but it continues to appear in forward sweep. So the selection of forward or backward sweep is determined by the focus of the design, suction performance, or cavitation instability. Further research is needed into how the secondary flow affects the development of cavity and cavitation instabilities.

For marine propellers, forward skew (in marine propellers it is more common to use the term skew instead of sweep) is not

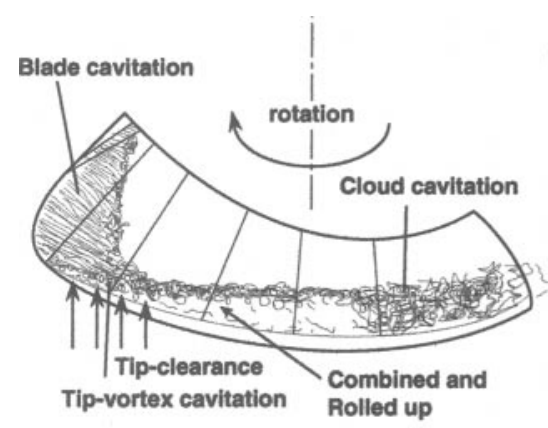

(a) Conventional Type

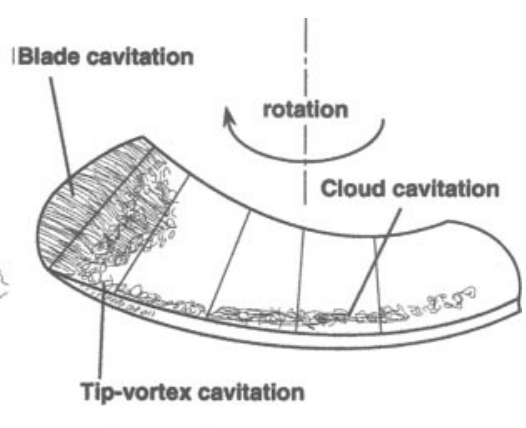

(b) Long-Lived Type

FIGURE 14

Comparison of cavitation pattern. 
usually used because of the structural problem and also because they must be capable of reverse operation. Backward skew is used to reduce the force of interaction with the hull's wake and also to reduce the hull's pressure pulsation. However, backward skew can lead to structural failure of the blade when reversing. This is why in recent applications, the inner radii have forward skew while the outer radii have backward skew like the one shown in Figure 8. Forward skew is generally used for propeller fans in air-conditioning units to reduce noise. For such types of fans, we usually have radially inward flow at the inlet and significant tip vortex. This prevents separation on the suction surface, much like the leading-edge vortex on delta wings, and the load on the blade can be increased and the rotating speed decreased.

\section{Integral Design of Inducers and Main Impellers}

Inducers are often designed separately from main impellers. There are several reasons for this:

1. For industrial pumps, inducers are prepared as an option to the main impeller.

2. To reduce the amount of overhang resulting from the mechanical design of the rotor system.

3. To get pressure recovery before the flow is introduced to the main impeller.

4. To provide a space where the cavitation in the inducer can collapse before entering the main impeller.

In spite of these facts, many early propellant pumps were integrated with the inducer as well as with separate inducers. Now many more CFD design tools and experimental methods are available to explore the design of the integrated approach, and it seems likely that this development will continue.

\section{Cavitation Instabilities and Design Criteria}

Various types of cavitation instabilities are reported by the panelists, and it is now becoming clear that there are various modes in addition to the classical cavitation surge and rotating cavitation modes. Although a two-dimensional cavitating flow stability analysis also predicts various modes, the correlation between the prediction and the observation is not yet clear. Modeling pump cavitation characteristics by using a transfer matrix is still useful for understanding and predicting a system's cavitation instabilities, including acoustic coupling, but only a limited number of systematic data are available, and we need to keep accumulating such data about a range of design variables. It is also important to correlate such data with internal flow. In this respect, it is proposed by a panelist to correlate the cavitation characteristics with the pressure gradient, focusing on the favorable effects of backflow. This leads to a new design criteria of increasing the slope of the pressure distribution at the throat. On the other hand, we do have three types of cavitation: blade surface, tip leakage, and backflow vortex cavitation. They should have important effects on cavitation but their separate effects are not very clear as yet.

So far, inducers have been designed principally on an empirical basis, as summarized by one of the panelists, and we depend on such empirical data for the basic engineering design. However, CFD is becoming a dependable tool to predict steady and even unsteady flow with cavitation, as presented in the panel. Experimental tools such as PIV provide detailed flow structure in pumps. These abilities were not available 25 years ago when one-dimensional instabilities such as POGO and cavitation surge were the major concerns. With these newer, more powerful tools, it is expected that we will be able to obtain explanations for the new instabilities reported in the panel and offer new methodologies for the design of stable and reliable inducers and inducer main-stage performance. One successful example of the application of CFD is presented by the panel; it focuses on reducing cavitation damage by employing a three-dimensional inducer design.

\section{CONCLUSIONS}

In addition to the three topics reported in the preceding section, the panel also discusses thermodynamic effects, especially for liquid hydrogen turbopumps, and the effects and control of foreign nuclei in turbopump tests. New cavitation instabilities are reported by space development agencies, showing the need for research to clarify the phenomena and to establish a design method that eliminates them. Empirical design methods for inducers are summarized; new design criteria that minimize cavitation instabilities are proposed; and an overview of cavitating marine propeller design is presented. CFD results are very promising, covering as they do predictions of suction performance and cavitation instabilities. Experimental fluid dynamics have already been applied to flows with simple geometry and are beginning to be applied to turbomachines. It is now necessary and possible to apply these powerful tools to the correlation of the detailed flow process with the global phenomena; the final goal is to establish design criteria for stable high-suction performance inducers. In conducting these efforts, we should not forget that the real causes often cannot be seen visually, as exemplified by cavitation surge, so flow modelings will continue to play an important role in the future. In any case, the panel has shown that we are now in a very interesting technical stage, and significant progress may be expected in the near future.

\section{In Memoriam}

We regret to say that the senior author, Marc Wegner, passed away on February 13, 2003. 

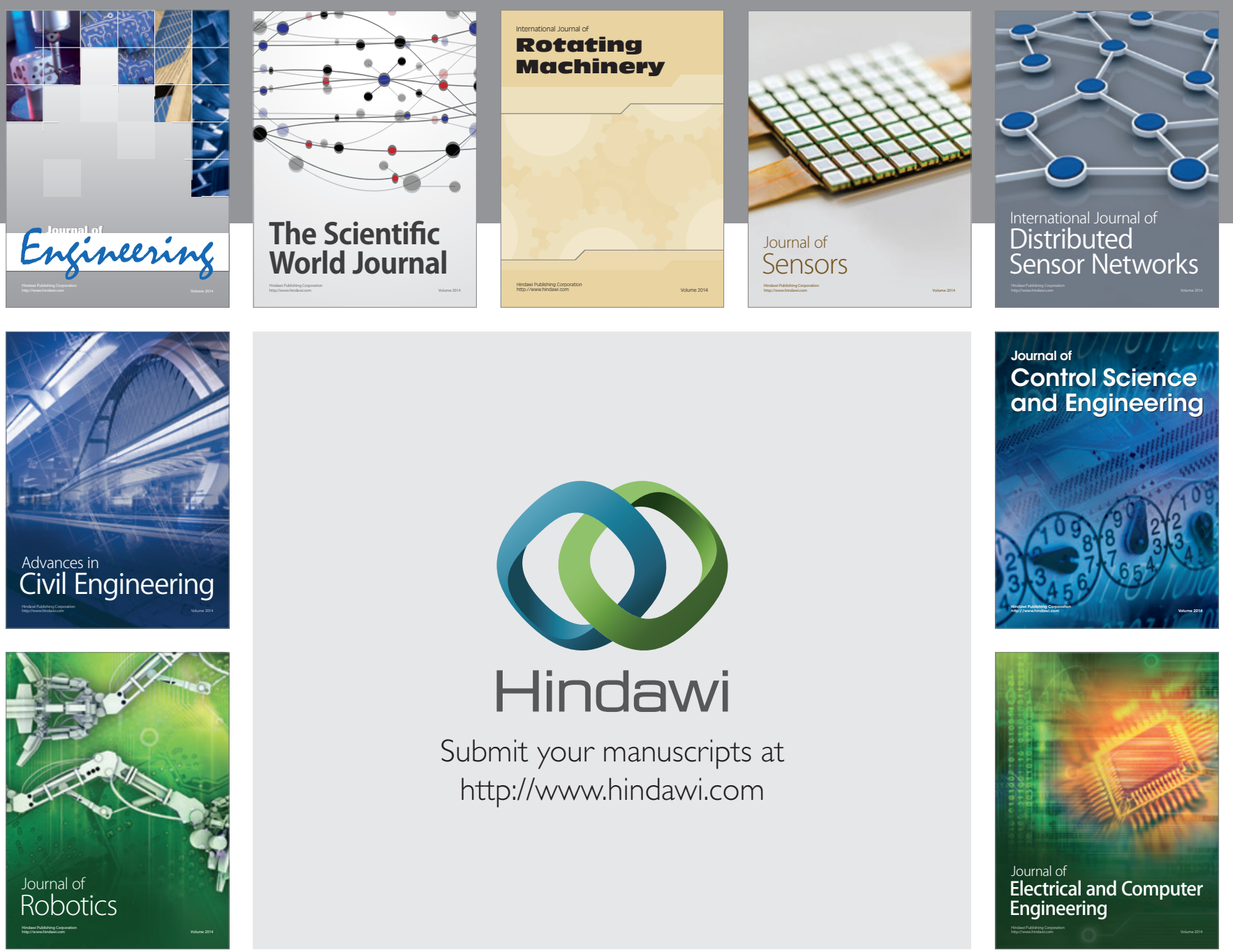

Submit your manuscripts at

http://www.hindawi.com
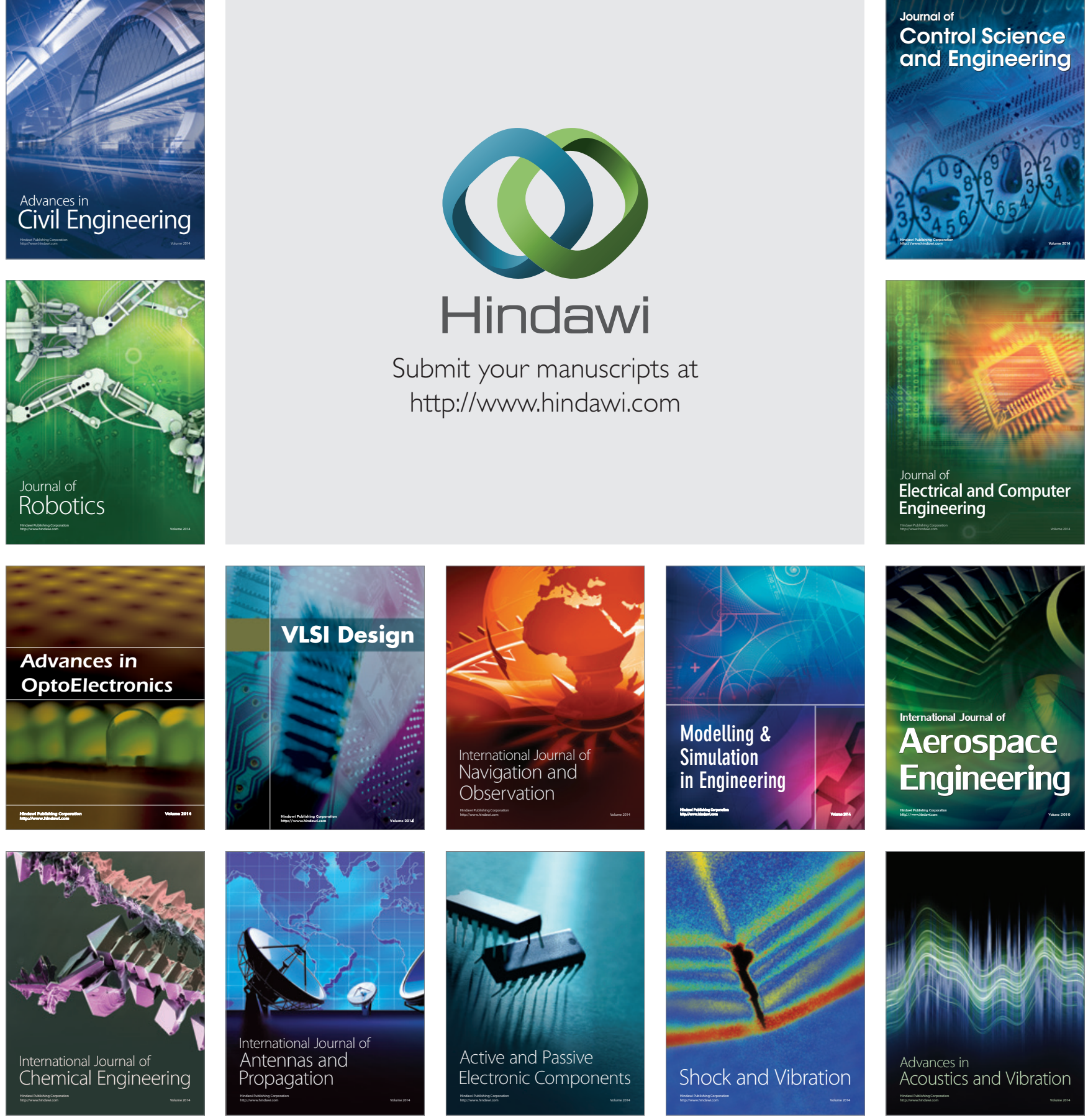